\title{
Epidemiology of urinary tract infection and antimicrobial resistance in a pediatric hospital in Nepal
}

\author{
Rabina Ganesh ${ }^{1,2}$, Dhiraj Shrestha ${ }^{2,3^{*}} \mathbb{D}$, Balkrishna Bhattachan ${ }^{1}$ and Ganesh Rai ${ }^{2}$
}

\begin{abstract}
Background: Urinary tract infection is an infection affecting infants and children. The aim of this study was to determine the etiology of urinary tract infection along with their antimicrobial resistance.

Methods: This cross-sectional study was conducted from June 2015 to January 2016 at Siddhi Memorial Hospital, Bhaktapur, Nepal. Urine samples were first cultured on cystine lactose electrolyte deficient agar and blood agar by semi-quantitative technique, and then incubated aerobically for $18-24 \mathrm{~h}$ at $37^{\circ} \mathrm{C}$. The identified bacterial isolates were tested for antimicrobial susceptibility by Kirby Bauer disc diffusion technique.

Results: Of 1599 urine samples, 12.3\% samples showed significant bacterial growth. E. coli (58.7\%) was the most common pathogen, followed by Klebsiella pneumoniae (22.5\%). Most of the isolates were resistant to ampicillin and co-trimoxazole, while least were resistant to amikacin and nitrofurantoin. Higher multi-drug resistance (61.9\%) was observed among isolates.
\end{abstract}

Conclusions: E. coli and Klebsiella spp. were predominant cause of pediatric urinary tract infection in children. Higher susceptibility observed against aminoglycosides and nitrofurans make these drugs suitable in emergency.

Keywords: Antimicrobial resistance, E. coli, Klebsiella spp., Nepal, Urinary tract infection

\section{Background}

Urinary tract infection (UTI) is any infection leading to an inflammatory response in the epithelium of the urinary tract [1]. UTIs affect both males and females of all ages. The occurrences of UTIs are higher in women, which are likely caused by anatomical differences, hormonal effects and behaviors [2]. The cases of UTI among Nepalese patients attending general hospitals ranges from 23.1 to $37.4 \%$ [3]. Bacteria are the common etiology of UTIs accounting more than $95 \%$ of the cases. Escherichia coli is the most common causative organisms of UTI and is solely responsible for more than $80 \%$ of UTI [4].

This study was conducted among children with symptoms of UTI visiting a tertiary healthcare in Nepal to

\footnotetext{
* Correspondence: hiraj.diamond@gmail.com

${ }^{2}$ Department of Microbiology, Shi-Gan International College of Science and Technology, Kathmandu, Nepal

${ }^{3}$ Department of Microbiology, Tri-Chandra Multiple College, Kathmandu, Nepal

Full list of author information is available at the end of the article
}

determine spectra of uropathogens, especially $E$. coli and Klebsiella spp., and their antimicrobial resistance (AMR). This would help the clinician in using appropriate antibiotics for the clinical management of UTI.

\section{Methods \\ Study design, area and sample population}

The cross-sectional study was conducted from June 2015 to January 2016 in Siddhi Memorial Hospital, Bhaktapur, Nepal. The Hospital is the only children hospital in the district and serves infants and children patients of the district and other nearby areas. The totals of 1599 urine samples were included in this study. The study populations were infants and children patients not exceeding 14 years age, seeking treatment at the hospital with symptoms of UTI. The symptoms included fever along with dysuria and/or loss of bladder control and/or lower back pain and/or cloudy or foul smelling urine. For infants and younger children symptoms included was fever and parental reporting of cloudy or foul 
smelling urine. The clean catch urine samples were collected in sterile container. In infants and non-toilet-trained children, a sterile foil bowl was placed underneath the genitalia for clean-catch urine collection. If this was not possible, a plastic bag was attached to genitalia for urine collection. In toilet-trained children, a clean-catch voided midstream urine sample was collected. In both cases the genitalia was cleaned beforehand to reduce contamination. Invasive techniques were avoided for urine collection. Children receiving antimicrobial drugs treatment and children undergoing bladder catheterization within $48 \mathrm{~h}$ were excluded in the study. The study was ethically approved by the Institutional Review Committee of Shi-Gan International College of Science and Technology (SICOST).

\section{Laboratory examinations of samples Macroscopic examination of urine}

Urine color was observed macroscopically immediately after collection for possible signs of contaminations.

\section{Processing of samples and identification of the isolates}

First of all, $1 \mu \mathrm{L}$ of all urine samples were streaked on cystine lactose electrolyte deficient (CLED) agar (HiMedia Pvt. Ltd., India) and blood agar (HiMedia Pvt. Ltd., India) by semi-quantitative method using calibrated loop. The plates were then incubated aerobically for 18 $48 \mathrm{~h}$ at $37^{\circ} \mathrm{C}$ and growths were observed. Positive culture result was considered for plates showing 100 or more colonies i.e. more than or equal to $10^{5}$ colony forming units $(\mathrm{CFU}) / \mathrm{ml}$ [5]. Identifications of isolates were done considering Gram's staining morphology, cultural characteristics and biochemical properties [6].

\section{Antimicrobial susceptibility testing}

All identified isolates of E. coli and Klebsiella spp. were tested for susceptibility against amikacin $(30 \mu \mathrm{g})$, ampicillin $(10 \mu \mathrm{g})$, cephalexin $(30 \mu \mathrm{g})$, cefixime $(10 \mu \mathrm{g})$, cefpodoxime $(30 \mu \mathrm{g}), \quad$ co-trimoxazole $(1.25 / 23.75 \mu \mathrm{g})$, nitrofurantoin $(300 \mu \mathrm{g})$, ofloxacin $(5 \mu \mathrm{g})$ (HiMedia Pvt. Ltd., India) using Kirby Bauer disc diffusion technique on Mueller-Hinton agar (HiMedia Pvt. Ltd., India). The results were interpreted using CLSI 2015 [7]. Quality control was accessed using $E$. coli ATCC 25922. Isolates resisting two or more classes of antimicrobial agents were considered multidrug resistant (MDR) [8].

\section{Data management and statistical analysis}

Data obtained were entered and managed in Microsoft Excel (version 2010, Microsoft Corporation, USA), and relation of variables were analyzed using ratio and percentage.
Table 1 Gender and age distribution of UTIs among children

\begin{tabular}{llll}
\hline Age group (years) & Male (\%) & Female (\%) & Total \\
\hline$\leq 1$ & $24(43.6)$ & $31(56.3)$ & 55 \\
$2-5$ & $33(36.6)$ & $57(63.3)$ & 90 \\
$6-9$ & $17(41.4)$ & $24(58.5)$ & 41 \\
$10-14$ & $3(27.2)$ & $8(72.7)$ & 11 \\
Total & $\mathbf{7 7 ( 3 9 . 1 )}$ & $\mathbf{1 2 0 ( 6 0 . 9 )}$ & $\mathbf{1 9 7}$ \\
\hline
\end{tabular}

\section{Results}

Among total 1599 urine samples processed, 929 (58.1\%) samples were collected from male children while 668 (41.8\%) samples from female children. Of 1599 samples, only $197(12.3 \%)$ samples were culture positive and hence UTI. Of 197 UTI cases, UTIs were higher in female children, i.e. 120 (60.9\%) compared to male children, i.e. 77 (39.1\%). UTIs were highest in age group 25 years (Table 1 ).

Rate of UTIs in inpatients and outpatients were similar (Table 2).

Total of seven different species of Gram negative bacteria were isolated. Among them, E. coli, 114 (57.8\%) was predominant followed by Klebsiella spp., 62 (31.4\%) (Fig. 1).

Most $K$. oxytoca isolates were resistant to cephalexin, $16(94.1 \%)$ and least were resistant to nitrofurantoin, 4 (23.5\%) and amikacin, 4 (23.5\%). Likewise, most K. pneumoniae isolates were resistant to ampicillin, 35 (77.8\%) and least were resistant to amikacin, 4 (8.9) (Fig. 2). Most E. coli isolates were resistant to ampicillin, 82 (71.9\%) and least were resistant to nitrofurantoin, 5 (4.4\%) (Fig. 3). Higher score of MDR, 109 (61.9\%), was observed among isolates (Table 3).

\section{Discussion}

UTIs are frequent in children and may incur adverse consequences [9]. Thus, reliable diagnosis of UTI in children is critical. In our study, only $12.3 \%$ of the 1599 urine samples yielded culture positive result. This warrants discordance of clinical and laboratory examination in the hospital. Laboratories choose one diagnostic threshold but there is no universally accepted threshold. We used the threshold of $10^{5} \mathrm{CFU} / \mathrm{ml}$ to determine culture positive result. This can miss lower bacterial counts. Higher threshold could have decreased the culture positive result. Lowering the threshold to $10^{4} \mathrm{CFU} / \mathrm{ml}$ can produce clinically valuable results [10].

Table 2 Distribution of UTIs in children

\begin{tabular}{llll}
\hline Types of patient & Total patients & UTI positive & $\%$ \\
\hline Inpatient & 159 & 20 & 12.6 \\
Outpatient & 1440 & 177 & 12.3 \\
Total & 1599 & 197 & 12.3 \\
\hline
\end{tabular}




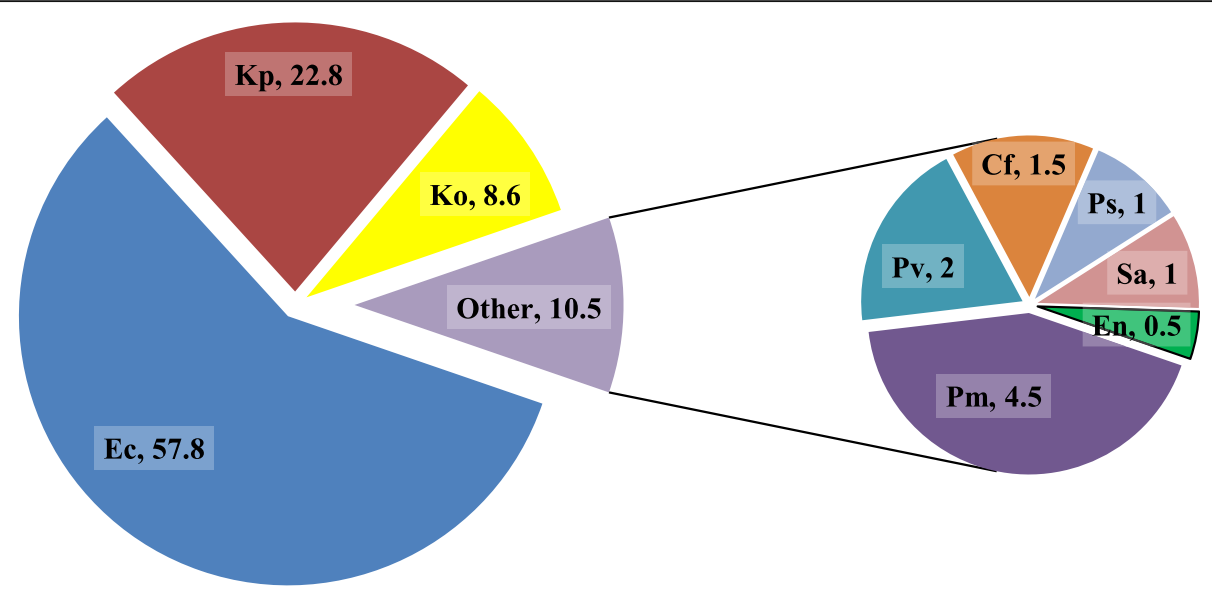

Fig. 1 Identified bacterial isolates in UTI patients (\%). Legends (as represented by different colors in pie diagram): Each color represent the fraction of the total isolated bacteria in percentage namely, $\mathrm{EC}=E$. coli, $\mathrm{Kp}=K$. pneumoniae, $\mathrm{Ko}=K$. oxytoca, $\mathrm{Pm}=P$. mirabilis, $\mathrm{Pv}=P . \mathrm{vulgaris}, \mathrm{Cf}=$ C. freundii, Ps = Pseudomonas spp., Sa $=$ S. aureus, En = Enterococcus spp.

The study population had higher male children compared to female children (male to female ratio 3:2). This was the involuntary recruitment bias. The other bias could be greater parental urge and/or greater doctors' preference in testing male children than female children. Also, this could be due to greater difficulty in clinically diagnosing male UTI. On contrast, UTIs were higher in female children as compared to male children (female to male ratio= 3:2). UTIs are more common in girls compared to boys due to shorter length of urethra [11]. Also, this could be due to better clinical diagnosis of UTI in girls than boys by doctors. Or this could be due to more likelihood of growth from specimens collected from females, perhaps due to higher rates of contamination of male specimens. This concords with other reports $[3,4,9,12]$. With the increase in age, female children showed considerable increase in UTI compared to male children. Majority of UTIs were seen in the ages below 6 years of age. Likewise, rate of UTIs was fairly similar in outpatients and inpatients.

Among seven identified Gram negative bacterial species, E. coli $(57.8 \%)$, was the predominant followed by Klebsiella spp. (31.4\%). This concords with other studies $[3,4,9,12,13]$. E. coli and K. pneumoniae have emerged as serious pathogens worldwide. UTI

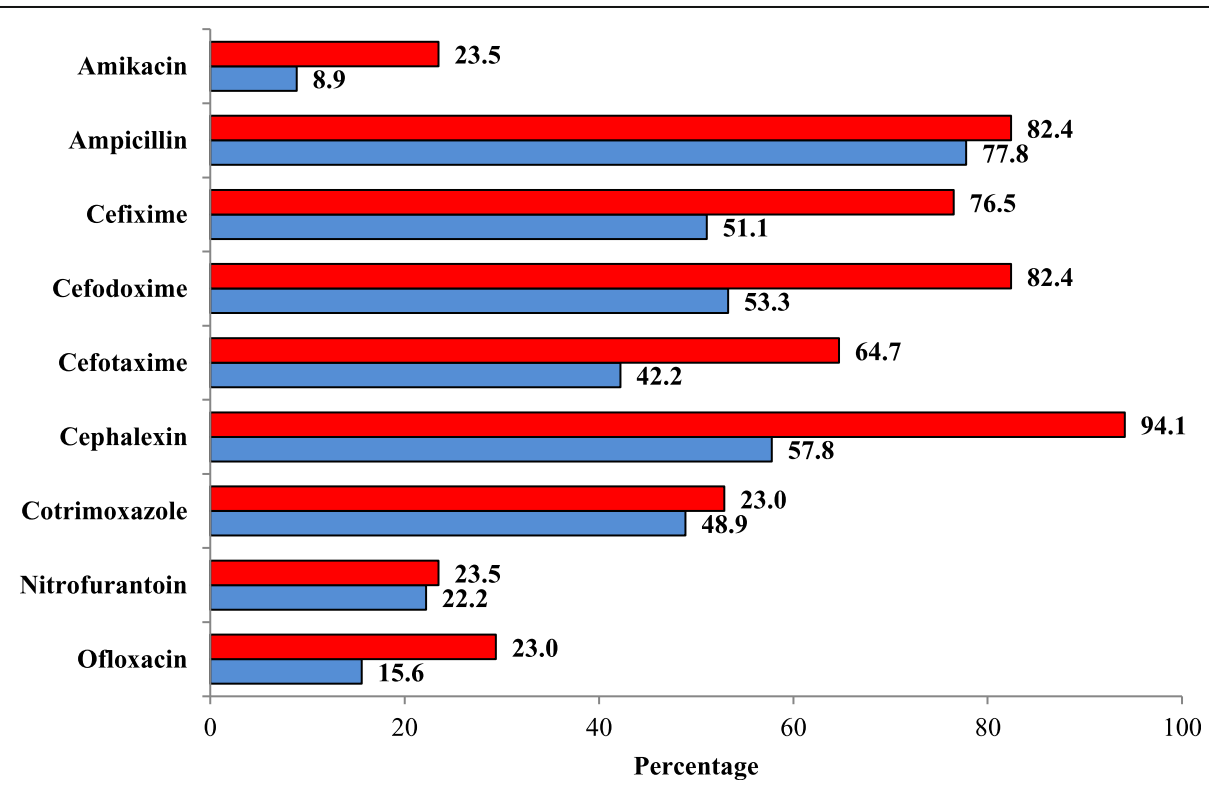

Fig. 2 Antibiotic resistivity of K. pneumoniae and K. oxytoca. Legends (as represented by two colors): Red color represents the resistivity percentage of $K$. oxytoca against the antibiotics. Blue color represents the resistivity percentage of $K$. pneumoniae against the antibiotics 


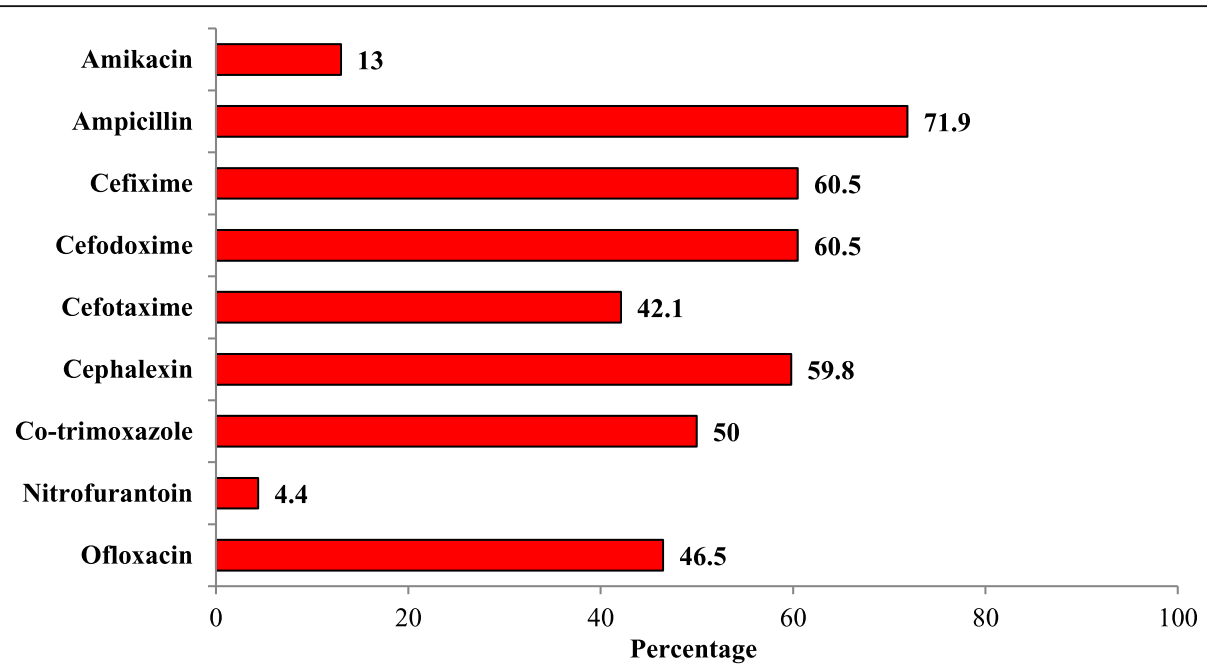

Fig. 3 Antibiotic resistivity of E. coli. Legends (as represented by red color): Red color represents the resistivity percentage of E. coli against the antibiotics

occurs when E. coli and/or other gastrointestinal tract flora enters urinary tract [14].

Antibiotics play crucial role in treating such infections as long as the etiological bacteria is susceptible to the antibiotic activity. Thus, determining accurate antibiotic susceptibility is essential in the clinical care of bacterial infections. Bacteria capable of acquiring resistance demands more attention [15]. Most scholars throughout the world are reporting increasing AMR.

Most children are treated empirically before laboratory findings to avoid complications [9]. Both E. coli and Klebsiella spp. showed high resistance to ampicillin. This concord with other studies [3, 4, 9, 12, 13]. Susceptibility of E. coli and $K$. pneumoniae against different cephalosporins was similar. About $60 \%$ isolates were resistance to cephalosporins thus cephalosporins can be treatment option but cannot be relied as reserve drug or empiric drug. But higher generations of cephalosporins could promise effectiveness as shown by lower resistance against cefotaxime, about $40 \%$. In contrast, $K$. oxytoca showed higher resistance to cephalosporins rendering ineffective for treatment. This further makes cephalosporins antibiotics unlikely for use as reserve 2nd line drug for treatment. Co-trimoxazole is preferred oral antibiotic for treatment of pediatric UTI but only half of the $E$. coli

Table 3 MDR among isolates

\begin{tabular}{lll}
\hline Bacteria & Total isolates & MDR isolates (\%) \\
\hline E. coli & 114 & $72(63.2)$ \\
Klebsiella pneumoniae & 45 & $26(57.8)$ \\
K. oxytoca & 17 & $11(64.7)$ \\
Total & 176 & $109(61.9)$ \\
\hline
\end{tabular}

and Klebsiella spp. isolates were susceptible. Thus, sulfonamide antibiotics cannot be relied solely for treatment. Ofloxacin was ineffective against half of the $E$. coli isolates and one-fifth of Klebsiella spp. isolates. This makes fluoroquinolones antibiotics unreliable 2nd line choice as well. Meanwhile, nitrofurantoin and amikacin were effective against most of the E. coli and Klebsiella spp. isolates. Thus, nitrofurans antibiotics can be relied as 1st line choice for empiric treatment. Nitrofurans is also the economical option as it is comparatively cheaper. Alternatively, aminoglycosides antibiotics can also serve as reasonable choice. These drugs can thus be reserved for emergency use. Higher score of MDR (61.9\%) among isolates underlines the higher risks associated with AMR and ask serious counter measures.

Inappropriate policies, poor surveillance, self-medication, poor diagnosis, poor quality of antibiotics, inadequate dose has all attributed to increase in AMR in recent years [16]. Inappropriate use of antibiotics in healthcare has increased the risk of AMR growth. This study was limited to phenotypic AMR detection excluding identification of different beta-lactamases producing bacteria. Genotyping characterization would provide further insight on AMR.

\section{Conclusions}

Our findings outline high AMR among common uropathogens in tertiary setting. Varying epidemiology of AMR with time and locales has hindered the effective management of infections. Since, our study was confined to single healthcare setting, only the broader and effective surveillance can establish AMR data for effective clinical management of such pathogens. Such AMR data is not available at present in this region thus, our 
findings can be referenced for the treatment of UTIs in children.

\section{Abbreviations}

AMR: Antimicrobial resistance; ATCC: American type culture collection; CFU: Colony forming unit; CLSI: Clinical Laboratory Standard Institute; MDR: Multidrug resistant; UTI: Urinary tract infection

\section{Acknowledgements}

We acknowledge children and their parents/guardians for supporting the study.

\section{Funding}

None.

\section{Availability of data and materials}

All data generated or analyzed during this study available in the public repository, figshare [https://doi.org/10.6084/m9.figshare.7359188.v1].

\section{Authors' contributions}

All authors made substantial contributions to the study. RG, BB and DS conceived and designed the study. RG collected sample, investigated and recorded the laboratory findings at the hospital. GR supervised and provided methodology for the study. RG and BB administered the project, reviewed literature, and drafted the manuscript. BB and DS compiled and curated data. DS helped in critical review and revision of the manuscript by compiling, formatting, editing and writing the final version of the manuscript. All authors read and approved the final manuscript.

\section{Ethics approval and consent to participate}

Institutional Review Committee of Shi-Gan International College of Science and Technology (SICOST), Kathmandu, approved this research. Written consent is not feasible for all subjects in Nepalese context since illiteracy is comparatively higher. Thus verbal consent was obtained from parents/guardians on the behalf of their children, as approved by ethical review committee, to omit the selection bias.

\section{Consent for publication}

Not applicable

\section{Competing interests}

The authors declare that they have no competing interests.

\section{Publisher's Note}

Springer Nature remains neutral with regard to jurisdictional claims in published maps and institutional affiliations.

\section{Author details}

${ }^{1}$ Department of Microbiology, Siddhi Memorial Hospital, Bhaktapur, Nepal. ${ }^{2}$ Department of Microbiology, Shi-Gan International College of Science and Technology, Kathmandu, Nepal. ${ }^{3}$ Department of Microbiology, Tri-Chandra Multiple College, Kathmandu, Nepal.

Received: 16 July 2018 Accepted: 16 April 2019

Published online: 14 May 2019

\section{References}

1. Nature, Macmillan Publishers Limited. Urinary tract infection. Available at: https://www.nature.com/subjects/urinary-tract-infection. Accessed 01/02/ 2016

2. Minardi D, d'Anzeo G, Cantoro D, Conti A, Muzzonigro G. Urinary tract infections in women: etiology and treatment options. Int J Gen Med. 2011;4: 333-43. Full Text

3. Rai GK, Upreti HC, Rai SK, Shah KP, Shrestha RM. Causative agents of urinary tract infections in children and their antibiotic sensitivity pattern: a hospital based study. Nepal Med Coll J. 2008;10(2):86-90. Full Text.

4. Nachimuthu R, Chettipalayam S, Velramar B, Kurumandur R, velu R Urinary tract infection and antimicrobial susceptibility pattern of extended spectrum beta lactamase producing clinical isolates. Adv Biol Res. 2008;2(5-6):78-82. Full Text.
5. Vandepitte J, Engbaek K, Piot P, Heuck CC, et al. Basic laboratory procedures in clinical bacteriology. 2nd ed. Geneva: World Health Organization; 2003. Full Text.

6. Versalovic J, Carroll KC, Funke G, Jorgensen JH, Landry ML, Warnock DW. (ed). Manual of clinical microbiology. 10th ed. American Society of Microbiology; 2011. http://www.asmscience.org/content/book/10.1128/ 9781555816728

7. Clinical and Laboratory Standards Institute (CLSI). Performance Standards for Antimicrobial Susceptibility Testing (M100-S25). 25th ed. Wayne, PA: Clinical and Laboratory Standards Institute; 2015.

8. Magiorakos AP, Srinivasan A, Carey RB, Carmeli Y, Falagas ME, Giske CG, Harbarth S, Hindler JF, Kahlmeter G, Olsson-Liljequist B, Paterson DL. Multidrug-resistant, extensively drug-resistant and pandrug-resistant bacteria: an international expert proposal for interim standard definitions for acquired resistance. Clin Microbiol Infect. 2012;18(3):268-81. Full Text.

9. Asokan GV, Vanitha A. Disaster response under one health in the aftermath of Nepal earthquake. J Epidemiol Glob Health 2017;7(1):91-96. [Full Text].

10. Montini G, Tullus, Hewitt I. Febrile urinary tract infections in children. N Engl Med 2011;365:239-250. [Full Text].

11. Doern CD, Richardson SE. Diagnosis of urinary tract infections in children. Kraft CS, ed J Clin Microbiol 2016;54(9):2233-2242. [Full Text].

12. Salvatore $S$, Salvatore $S$, Cattoni E, Siesto G, Serati M, Sorice P, Torella M. Urinary tract infections in women. Eur J Obstet Gynecol Reprod Biol 2011; 156(2):131-136. [Full Text].

13. De Miranda ÉJP, De Oliveira GSS, Roque FL, Dos Santos SR, Olmos RD, Lotufo PA. Susceptibility to antibiotics in urinary tract infections in a secondary care setting from 2005-2006 and 2010-2011, in São Paulo, Brazil: data from 11,943 urine cultures. Rev Inst Med trop S Paulo. 2014; 56:313-24. Full Text.

14. McLellan LK, Hunstad DA. Urinary tract infection:pathogenesis and outlook. Trends Mol Med. 2016:22(11):946-57. Full Text.

15. Jorgensen $\mathrm{JH}$, Ferraro MJ. Antimicrobial susceptibility testing: a review of general principles and contemporary practices. Clin Infect Dis. 2009;49(11): 1749-55. Full Text.

16. Siegel JD, Rhinehart $\mathrm{E}$, Jackson $\mathrm{M}$, Chiarello L. Management of multidrugresistant organisms in health care settings, 2006. Am J Infect Control. 2007; 35(10):S165-93. Full Text.
Ready to submit your research? Choose BMC and benefit from:

- fast, convenient online submission

- thorough peer review by experienced researchers in your field

- rapid publication on acceptance

- support for research data, including large and complex data types

- gold Open Access which fosters wider collaboration and increased citations

- maximum visibility for your research: over $100 \mathrm{M}$ website views per year

At BMC, research is always in progress.

Learn more biomedcentral.com/submissions 